\title{
Chinese Herbal Medicine Combined Conventional Chemotherapy Regimens in Advanced Recurrent Breast Mammary Carcinoma
}

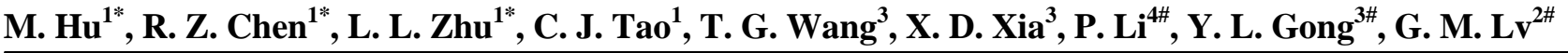 \\ ${ }^{1}$ Graduate School, Medical College, Southeast University, Nanjing, China; ${ }^{2}$ Department of Nursing Education, Jiangsu Jiankang \\ Vocational College, Nanjing, China; ${ }^{3}$ Department of Oncology, Nanjing 1st Hospital, Nanjing, China; ${ }^{4}$ Department of Gastroen- \\ terology, Changhai Hospital, Shanghai, China. \\ Email: "Lvguangmei@aliyun.com, ${ }^{\#}$ Pingli1965@163.com, " gongyongling26@163.com
}

Received November $7^{\text {th }}, 2013$; revised December $28^{\text {th }}, 2013$; accepted December $5^{\text {th }}, 2013$

Copyright (C) 2013 M. Hu et al. This is an open access article distributed under the Creative Commons Attribution License, which permits unrestricted use, distribution, and reproduction in any medium, provided the original work is properly cited. In accordance of the Creative Commons Attribution License all Copyrights (C) 2013 are reserved for SCIRP and the owner of the intellectual property M. Hu et al. All Copyright (C) 2013 are guarded by law and by SCIRP as a guardian.

\begin{abstract}
Purpose: Aiming at starting the ball rolling and contributing humble effort to promoting traditional Chinese medicine, we report one successful case of "GP" regimen of combining chemotherapy with traditional Chinese medicine decoction ("lung description") for breast cancer with lung and bone metastases. Patients and Methods: A second-line chemotherapy regimen of GP (cisplatin + gemcitabine) was applied. In the meantime, we administered Chinese herbal medicine (Fei Decoction, mixed with a variety of effective herbal components) to help her recover from the poor condition. Results: The tumor markers (CEA, CA15-3) had dramatically decreased to the normal range. Both lung and bone metastatic sites reduced according to CT and ECT imaging, and this patient felt free from the complaint of pulmonary and cardiac discomfort. The quality of life has been greatly improved, and the PFS (Progression-Free-Survival) and TTP (Time-to-Progression) have been prolonged from the onset to date. Conclusions: In the course of this combined treatment, it has been shown that Chinese herbal medicine played an important role in the therapy of breast mammary carcinoma. Therefore, Chinese herb may turn out to be an additional choice with its better benefits and tolerability in the treatment of recurrent breast mammary carcinoma.
\end{abstract}

Keywords: Breast Mammary Carcinoma; Chemotherapy; Chinese Medicine

\section{Introduction}

The incidence of breast cancer has increased because of the reduced mortality resulting from improved treatments and the development of advanced tools for the early detection of cancerous lesions [1]. Data released by China Cancer Research Foundation showed that breast mammary carcinoma might be the first leading cause of death from cancer of women in metropolis such as Shanghai, Beijing and Guangzhou. In Shanghai, the situation is more serious, for there are currently about 40,000 patients suffering from breast cancer, with the new cases rate being at 10/129 each day. Among these, 20\% to 30\% of breast cancer patients are accompanied with "black

\footnotetext{
${ }^{*}$ R.Z. Chen, L.L. Zhu and M. Hu contributed equally to this work.

${ }^{\#}$ Corresponding authors.
}

butterfly" known as HER2 positive breast cancer, whose prognosis is dismay in one disaster after another [2,3].

Thus, more patients with advanced recurrent breast mammary carcinoma are now encountered. Efforts to develop more effective drugs for treatment of recurring breast mammary carcinoma have been intensified.

Chinese medicine is a medical system that is capable of treating a very wide range of conditions. Together with acupuncture, massage, and food cures, they form the four distinct methods of treatment in traditional Chinese medicine (also known as TCM or T.C.M.). Today, TCM still plays a major part of health care in China, and is provided in state hospitals alongside with Western medicine [4]. Yet there are basic differences between Western medicine and TCM. Western medicine focuses more on the treatment of symptoms while TCM focuses more on 
the causes of those symptoms [5].

It has become an indisputable fact that the treatment of recurrent breast mammary carcinoma in advanced stage is characterized by poor clinical efficacy and adverse outcome, and the therapy efficacy of the combination treatment of traditional Chinese medicine and western medicine for this breast mammary carcinoma is to be further evaluated by the evidence-based medicine [6]. Currently, the combination treatment of chemotherapy and traditional Chinese medicine for the advanced recurrent breast mammary carcinoma has rarely been reported [7]. Hence, aiming at starting the ball rolling and contributing humble effort to promoting traditional Chinese medicine, we report one successful case of "GP" regimen of combining chemotherapy with traditional Chinese medicine decoction in Figure 1, "Fei formulary" for breast mammary carcinoma with lung and bone metastases.

\section{Case Presentation}

A 46-year-old Chinese woman with a postmenopausal history was presented to a local neighborhood clinic because of an abnormal mass in her right breast mammary gland seen on a molybdenum target radiography taken during an annual medical checkup. A chest computed tomography (CT) scan demonstrated a solitary breast cancer with an irregular border and a spiculated formation in her right breast gland, which had enlarged right axillary and mediastinal lymph nodes (LNs), suggesting invasive breast adenocarcinoma $\left(\mathrm{T}_{3} \mathrm{~N}_{2} \mathrm{M}_{0}\right)$ in Sep 2010. She was subsequently referred to local hospital for a radical surgical resection of her right breast cancer on Nov 16, 2011. Microscopic examination of the removal specimens showed locally advanced low-differentiated adenocarcinoma invaded gland ducts and lymph nodes (9/9), immunohistochemistry showed: ER++, PR++, Cerb2 + , which indicates stage III breast mammary carcinoma. Postoperative recovery is well, three cycles of postoperative chemotherapy (docetaxel and epi-ADM) and radiotherapy (DT:55Gy) were administered, and the final chemoradiotherapy was performed on Feb7, 2011, thereafter tamoxifen sequential endocrine therapy was administered.

$\begin{aligned} 1 & \text { semen coicis } \\ 2 & \text { peach kemel } \\ 3 & \text { Chinese wax gourd kernel } \\ 4 & \text { raw astragalus membranaceus } \\ 5 & \text { liliumbrownie } \\ 6 & \text { cortex albiziae } \\ 7 & \text { tuber of dwarf lilyturf } \\ 8 & \text { rhizoma bletillae } \\ 9 & \text { bulbus fritillariae cirrhosae } \\ 10 & \text { almond } \\ 11 & \text { platycodon grandiflorum } \\ 12 & \text { houttuyniacordata } \\ 13 & \text { radix glehniae } \\ 14 & \text { cynanchumatratum } \\ 15 & \text { liquorice } \\ 16 & \text { fried rice sprout } \\ 17 & \text { fried malt sprout }\end{aligned}$

Fei Decoction 1

$\begin{aligned} 1 & \text { radix glehniae } \\ 2 & \text { herba patriniae } \\ 3 & \text { sargent gloryvine } \\ 4 & \text { semen coicis } \\ 5 & \text { radix scutellariae } \\ 6 & \text { radix paeoniae rubra } \\ 7 & \text { cortex moutan } \\ 8 & \text { Chinese wax gourd kemel } \\ 9 & \text { pericarpium arecae } \\ 10 & \text { honeysuckle } \\ 11 & \text { medicated leaven } \\ 12 & \text { rhizoma imperatac } \\ 13 & \text { Chinese violet } \\ 14 & \text { bulbus fritillariae cirnhosae } \\ 15 & \text { rhizoma bletillae } \\ 16 & \text { lilium brownie } \\ 17 & \text { liquorice }\end{aligned}$

Fei Decoction 4

$\begin{array}{lrrl}10 \mathrm{~g} & 1.2 & 1 & \text { semen coicis } \\ 10 \mathrm{~g} & 1 & 2 & \text { peach kemel } \\ 10 \mathrm{~g} & 1.2 & 3 & \text { Chinese wax gourd kemel } \\ 10 \mathrm{~g} & 2.5 & 4 & \text { raw astragalus membranaceus } \\ 10 \mathrm{~g} / & 1 & 5 & \text { lilium brownie } \\ 10 \mathrm{~g} & 1 & 6 & \text { cortex albiziae } \\ 10 \mathrm{~g} & 1 & 7 & \text { tuber of dwarf lilyturf } \\ 10 \mathrm{~g} & 1 & 8 & \text { thizoma bletillae } \\ 10 \mathrm{~g} & 1 & 9 & \text { bulbus fritillariae cirnosae } \\ 10 \mathrm{~g} & 1 & 10 & \text { almond } \\ 10 \mathrm{~g} / & 0.6 & 12 & \text { flatycodon grandiflorum } \\ 10 \mathrm{~g} & 1.2 & 13 & \text { houttus aurantii } \\ 10 \mathrm{~g} & 1 & 14 & \text { radix glehniae } \\ 10 \mathrm{~g} & 1 & 15 & \text { cynanchum atratum } \\ 10 \mathrm{~g} & 0.6 & 16 & \text { fried rice sprout } \\ 10 \mathrm{~g} / & 1 & 17 & \text { liquorice } \\ 10 \mathrm{~g} & 1 & 18 & \text { fried malt sprout } \\ & & 19 & \text { raw rhubarb }\end{array}$

Fei Decoction 2

$\log /$
$\log /$
$10 g /$
$10 g /$
$\log /$
$10 g /$
$10 g /$
$10 g /$
$10 g /$
$10 g /$
$\log /$
$10 g /$
$10 g /$
$\log /$
$10 g /$
$10 g /$
$10 g /$

$10 g^{\prime}$
$10 g^{\prime}$
$10 g^{\prime}$
$10 g^{\prime}$
$10 g^{\prime}$
$10 g^{\prime}$
$10 g^{\prime}$
$10 g^{\prime}$
$10 g^{\prime}$
$10 g^{\prime}$
$10 g^{\prime}$
$10 g^{\prime}$
$10 g^{\prime}$
$10 g^{\prime}$
$10 g^{\prime}$
$10 g^{\prime}$
$10 g^{\prime}$
$10 g^{\prime}$
$10 g^{\prime}$

$\begin{array}{lr}10 \mathrm{~g} / & 1.5 \\ 10 \mathrm{~g} / & 1 \\ 10 \mathrm{~g} / & 1 \\ 10 \mathrm{~g} / & 1.2 \\ 10 \mathrm{~g} / & 1.2 \\ 10 \mathrm{~g} / & 1 \\ 10 \mathrm{~g} / & 1 \\ 10 \mathrm{~g} / & 1.5 \\ 10 \mathrm{~g} / & 1 \\ 10 \mathrm{~g} / & 1 \\ 10 \mathrm{~g} / & 1 \\ 10 \mathrm{~g} / & 1 \\ 10 \mathrm{~g} / & 1 \\ 10 \mathrm{~g} / & 1 \\ 10 \mathrm{~g} / & 2 \\ 10 \mathrm{~g} / & 1 \\ 10 \mathrm{~g} / & 0.5\end{array}$

Fei Decoction 3

$\log /$
$\log /$
$\log /$
$\log /$
$\log /$
$\log /$
$\log /$
$10 g /$
$10 g /$
$\log /$
$\log /$
$\log /$
$\log /$
$\log /$
$\log /$
$\log /$
$\log /$
$10 g /$
$\log /$
$\log /$
$10 g /$

\begin{tabular}{|c|c|c|c|}
\hline 3 & 1 & rhizoma phragmitis & $10 \mathrm{~g} /$ \\
\hline $\begin{array}{r}1.2 \\
3\end{array}$ & 2 & semen coicis & $10 \mathrm{~g}$ \\
\hline 3 & 3 & Chinese wax gourd kemel & $10 \mathrm{~g}$ \\
\hline $\begin{array}{r}2.5 \\
1\end{array}$ & 4 & artemisia apiacea & $10 \mathrm{~g}$ \\
\hline 3 & 5 & peach kemel & $10 \mathrm{~g}$ \\
\hline $\begin{array}{r}1.2 \\
3\end{array}$ & 6 & lilium brownii & $10 \mathrm{~g} /$ \\
\hline 3 & 7 & turtle shell & $10 \mathrm{~g} /$ \\
\hline $\begin{array}{r}1.2 \\
1\end{array}$ & 8 & cortex albiziae & $10 \mathrm{~g} /$ \\
\hline 3 & 9 & radices stellariae dichotomae & $10 \mathrm{~g} /$ \\
\hline $\begin{array}{r}1 \\
1.2\end{array}$ & 10 & cynanchum atratum & $10 \mathrm{~g} /$ \\
\hline 2 & 11 & contex lycii radicis & $10 \mathrm{~g}$ \\
\hline $\begin{array}{r}1 \\
1.2\end{array}$ & 12 & platycodon grandiflonum & $10 \mathrm{~g} /$ \\
\hline 1.2 & 13 & dried rhizome of rehmannia & $10 \mathrm{~g} /$ \\
\hline $\begin{array}{l}3 \\
1\end{array}$ & 14 & liquorice & $10 \mathrm{~g}$ \\
\hline
\end{tabular}

Fei Decoction 6

Figure 1. Formulary of Fei Decoction 1 - 6, mixed with a variety of effective herbal components. Sig: decoct medicinal herbs in $300 \mathrm{ml}$ water per day, $150 \mathrm{ml}$, bid (9:00/am, 15:00 pm). 
By the end of 2011, she began to complain of dry cough, chest pain, dyspnea, palpitations, headedness, weakness and fatigue, and those symptoms went from bad to worse. So this patient was referred to our medical college and hospitalized in the oncological department in Jan, 2012. Conventional laboratory data showed iron deficiency anemia and elevated levels of carcinoembryonic antigen (CEA: $8.45 \mathrm{ng} / \mathrm{ml}$ ) and carbohydrate antigen (CA15-3:282.80 U/ml). Systemic evaluation, including $\mathrm{CT}$ and ECT imaging, showed evidence of multiple pulmonary, mediastinal LNs, and skeletal metastatic lesions, accompanied with complications consisted of pleural effusion, arrhythmia, pericardial effusion and low cardiac output syndrome, etc. Upon diagnosis it was confirmed as advanced recurrent breast mammary carcinoma $\mathrm{T}_{4} \mathrm{~N}_{2} \mathrm{M}_{1}$ ). Assessment of HER2 tumor status by fluorescence in situ hybridization (FISH) assay was judged as being an inappropriate case for trastuzumab target treatment, due to its negative result. In accordance with our patient's request, she received the treatment of secondline chemotherapy regimen of GP with cisplatin $(20 \mathrm{mg}$, $\mathrm{d} 1-5)$ plus gemcitabine $(1.4 \mathrm{~g}, \mathrm{~d} 1,8)$ every four weeks on the basis of the available Chinese clinical practice guidelines in oncology. Chemoradiotherapy was not chosen as a treatment option because of the requirement for an extended radiation field. In the meantime, we administered Chinese herbal medicine (Figure 1: Fei Decoction 1 - 2, mixed with a variety of effective herbal components) to get far better benefits and tolerability.

Effect of Fei Decoction 1: tonifying Yang and benefiting Qi, including inducing diuresis to alleviate edema, clearing away lung heat and expelling abscess. Effect of Fei Decoction 2: strengthening purgation and detoxification based on Fei Decoction 1.

After 2 cycles of chemotherapy plus Chinese traditional treatment, the tumor marker dramatically decreased (CEA: $8.45 \rightarrow 1.63 \mathrm{ng} / \mathrm{ml}$, CA15-3: $282.80 \rightarrow$ $58.89 \mathrm{U} / \mathrm{ml})$. Both lung and bone metastatic sites reduced in size, and judged as stable disease(SD) using the Response of Evaluation Criteria in Solid Tumors criteria, with the patient feeling free from the complaint of pulmonary and cardiac discomfort. Along with this favorable outcome, our patient received a further four cycles chemotherapy of GP plus Chinese herbal medicine (Figure 1: Fei Decoction 3 - 5). Effect of Fei Decoction 3: strengthening the efficacy of nourishing Yin and moistening lung based on Fei Decoction 1. Effect of Fei Decoction 4: strengthening the efficacy of clearing away heat and detoxifying, dissipating stasis and alleviating pain based on Fei Decoction 1. Effect of Fei Decoction 5: strengthening the efficacy of clearing away lung heat and reducing phlegm, relieving swelling and alleviating pain based on Fei Decoction 1.
In the course of chemotherapy, our patient also exhibited grade 3 - 4 leukopenia and neutropenia, which were dealt with clinical antibiotics (igecycline, $50 \mathrm{mg}, \mathrm{iv}, \mathrm{q} 12 \mathrm{~h}$, Hisun-Pfizer) and G-CSFPegfilgrastim, jinyouli, $6.0 \mathrm{mg}$, H, st, China Shijiazhuang Pharmaceutical Company), with the normal result of blood routine test after treatment (WBC: $0.9 \times 10^{9} / \mathrm{L} \rightarrow 6.3 \times 10^{9} / \mathrm{L}$ ). However, the treatment was successfully administered in the case of the breast cancer, with mediastinal LNs reduced, and was judged as a PR after six cycles of treatment. The final time of scheduled chemotherapy was July 15, 2012. One month after the last combination therapy, chest $\mathrm{CT}$ and skeletal ECT revealed no regrowth of pulmonary lesion and vertebral invasion, and deminished mediastinal LNs and pleural effusion, resulting in stable disease with a 6-month progression-free survival. The tumor marker (CEA, CA153) dramatically decreased to the normal range, and the quality of life has been greatly improved.

So the patient was discharged from our hospital after recovery, and received a further maintenance monotherapy with Chinese herbal medicine (Figure 1: Fei Decoction 6). Effect of Fei Decoction 6: strengthening the efficacy of clearing away lung heat and reducing fire, relieving swelling and alleviating pain based on Fei Decoction 1. A follow-up was scheduled with her outpatient service doctor every 6 month, including interval history, physical examination, conventional laboratory test, determination of tumor markers, chest $\mathrm{CT}$ and skeletal ECT. Follow-up results showed that the tumor marker (CEA, CA153) level finally came back to the normal range (Figure 2). Both lung and LNs metastatic sites reduced according to CT (Figure 3). Yet it came as a surprise to find an effect of diminishing the bone lesions by ECT imaging (Figure 4), and perfectly normal ECG. Our patient is still alive 37 months after diagnosis, and further Chinese traditional treatment is planned including both decoction and powder for oral taking. We managed to have prolonged the PFS (Progression-Free-Survival) and TTP (Time-to-Progression) from the onset to date.

\section{Discussion}

Chinese breast cancer patients see a doctor in an average age of 48.7 years old, among whom more than a third are diagnosed between 40 and 49 . The incidence of breast cancer has a trend of "urbanization" and "high-end crowd" [8]. Although China is located in the low area of breast cancer, the incidence has been increasing significantly in recent years, about 470,000 patients to date [9]. Those therapeutic effect and prognosis of recurrent or metastatic breast cancer is far from satisfactory. The overall concept of Chinese traditional medicine is based upon the theory that deficiency of vital "Qi" with prevalence of pathogenic "Qi" is the root cause of diseases and 


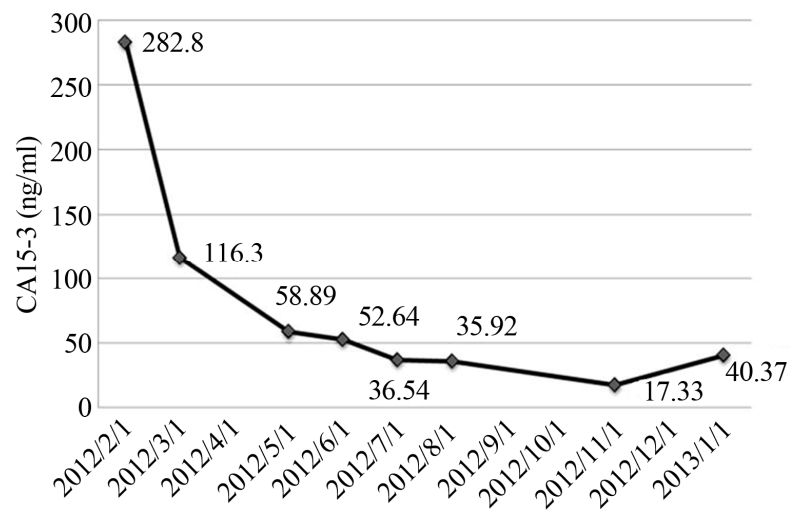

Figure 2. Changes of CA15-3 in the course of treatment.

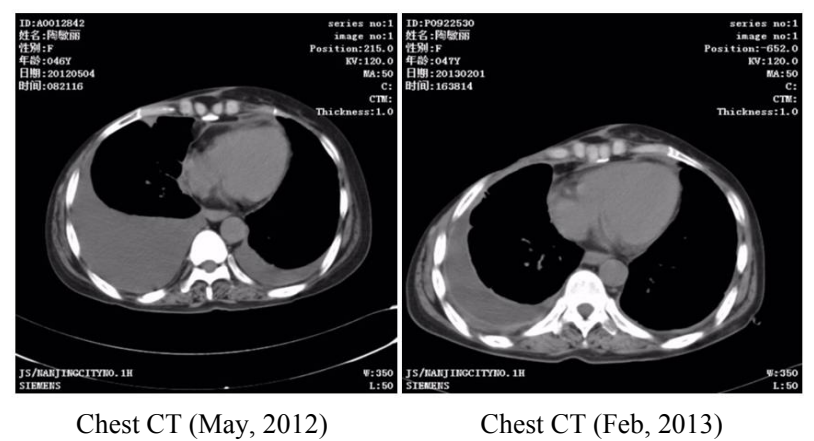

Figure 3. Comparison of chest CT before and after treatment.

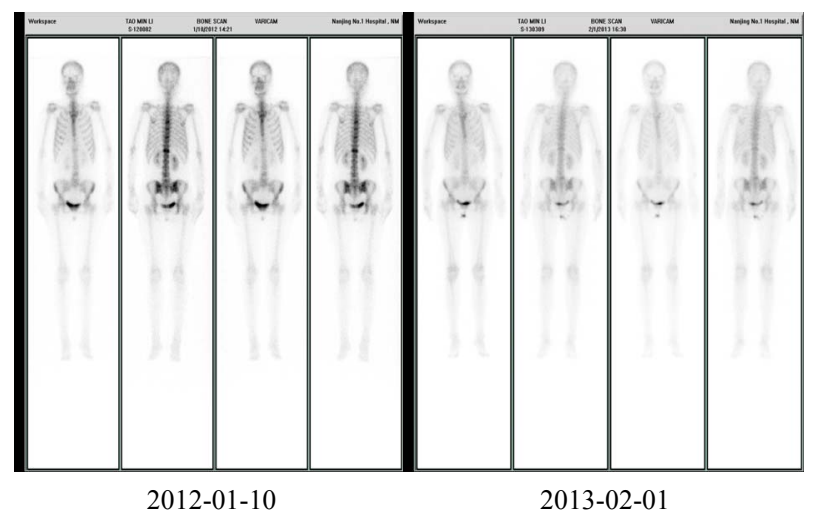

Figure 4. Comparison of ECT before and after treatment.

syndromes. It is believed that weakened body resistance coupled with prevailing pathogenic factors should be the fundamental cause in the initiation and development of tumor. Chinese traditional medicine clinical treatment aims to take into consideration a patient's whole condition, including body and mental condition, rather than confines to the cancer lesion itself [10]. Distinguished prescriptions should be made out to individual patients, so as to increase energy and heighten sense of well-being, reduce the side effect of radiation and chemotherapy, refresh marrow function and improve appetite and sleep
[11]. The research of Chinese traditional medicine anti-tumor effect has made great achievements in recent years [12]. It should be guided by the theory of Chinese traditional medicine to practice in oncology. With the development of science, it has obtained a new understanding of etiology, pathogenesis, and treatment of the tumor for human beings, who will pay a great deal of attention to the molecular mechanism of the anti-tumor effect in traditional Chinese medicine.

Principal, assistant, complement and mediating guide constitute the fundamental elements of traditional Chinese medicine medicinal prescription. Our case showed the efficacy of chemotherapy regimen GP plus Chinese herbal medicine Fei decoct in the treatment of advanced recurrent breast cancer. The followings is detailed explanation of prescription mentioned above.

Efficacy of Decoction 1: tonifying Yang and benefiting Qi, including inducing diuresis to alleviate edema, clearing away lung heat and expelling abscess.

Prescription analysis: Principal: in the description, astragalus membranaceus, tonifying Yang and benefiting $\mathrm{Qi}$, inducing diuresis to alleviate edema, detoxifying and expelling abscess; Assistant: Chinese wax gourd kernel, being sweet in taste and cold in nature and clearing away lung heat, reducing phlegm and expelling abscess; houttuynia cordata, being acrid in taste and cold in nature and distributing to lung, clearing away heat and detoxifying, eliminating carbuncle and expelling abscess, inducing diuresis for treating strangurtia. Complement and mediating guide: peach kernel, removing blood stasis, semen coicis, clearing away lung heat and expelling abscess; platycodon grandiflorum and almond, ascending and descending respectively to open lung energy, reduce phlegm and expel abscess; lilium brownii, radix Glehniae and tuber of dwarf lilyturf, being sweet in taste and cold in nature and moistening lung, nourishing Yin and clearing away heat; bulbus fritillariae cirrhosae, reducing phlegm and moistening lung to strengthen the efficacy; cortex albiziae, removing blood stasis and eliminating carbuncle, resolving stagnation for tranquilization, capable of distributing to liver and lung; cynanchum atratum, being bitter in taste and cold in nature, eliminate deficiency heat and treating ulcer and pyogenic infections; rice, malt sprouts, soothing liver and relieving depression, invigorating spleen and appetizing, promoting secretion and nourishing Yin and helping stomach Qi in developing; rhizoma bletillae, stopping bleeding and eliminating carbuncle, removing necrotic tissue and promoting granulation; and liquorice, moderating the property of the mentioned herbs.

Efficacy of Decoction 2: strengthening purgation and detoxification based on description 1 .

Prescription analysis: Principal, assistant, comple- 
ment and mediating guide are the same as those in the previous prescription analysis. Compared with the previous prescription, add fructus aurantii, relieving stuffiness of chest and regulating Qi, accompanied by raw rhubarb, for strengthening the efficacy of relieving constipation and relaxing bowels, eliminating carbuncle and dispersing swelling.

Efficacy of Decoction 3: strengthening the efficacy of nourishing Yin and moistening lung based on description 1.

Prescription analysis: Principle: radix rehmanniae preparata, nourishing Yin and enriching blood; dried rhizome of rehmannia, clearing away heat and cooling blood; radix scrophulariae, being salty in taste and cold in nature, helping the two medicines above in nourishing Yin and strengthening renal Yin and clearing away deficiency fire. Assistant: lilium brownii, radix Ophiopogonis, asparagus fern, distributing to lung, nourishing Yin and clearing away heat, moistening lung to arrest cough; bulbus fritillariae cirrhosae, being slight cold in nature, reducing phlegm and moistening lung; radix stemonae, tussilago farfara, being warm in nature, warming lung and reducing phlegm, moistening lung to arrest cough; the three medicines above are combined, mildly regulating cold and heat, greatly strengthening the efficacy of reducing phlegm and moistening lung. Complement: astragalus membranaceus, tonifying Yang and benefiting Qi, inducing diuresis to alleviate edema, detoxifying and expelling abscess; poria cocos, rizoma atractylodis macrocephalae and liquorice, being combined for warming middle-Jiao and tonifying deficiency, helping spleen and stomach in developing and upwards transferring nutrients to lung; cynanchum atratum, being bitter in taste and cold in nature, eliminating deficiency heat and treating ulcer and pyogenic infections; rhizoma bletillae, stopping bleeding and eliminating carbuncle, removing necrotic tissue and promoting granulation; pseudo-ginseng, being warm in nature, stopping bleeding and dissipating stasis, reducing swelling and alleviating pain, wherein Yin and Yang are united to strengthen the bleeding stopping efficacy.

Efficacy of Decoction 4: strengthening the efficacy of clearing away heat and detoxifying, dissipating stasis and alleviating pain based on description 1 .

Prescription analysis of decoction: Principle: herba patriniae, clearing away heat and detoxifying, eliminating carbuncle and expelling abscess, dissipating stasis and alleviating pain. Assistant: Chinese violet, honeysuckle, clearing away heat and detoxifying, cooling blood and eliminating carbuncle; sargent gloryvine, clearing away heat and detoxifying, promoting blood circulation and alleviating pain, removing periappendicular abscess and stomachache. Complement: semen coicis, Chinese wax gourd kernel, clearing away lung heat and expelling abscess; bulbus fritillariae cirrhosae, reducing phlegm and moistening lung, softening hard lumps and dispelling nodes; lilium brownii, being sweet in taste and cold in nature and moistening lung, nourishing Yin and clearing away heat; radix bupleuri, radix scutellariae, clearing away stagnated fire of liver and gall, harmonizing ShaoYang; cortex moutan, radix paeoniae rubra together with radix bupleuri, radix scutellariae, soothing liver-gallbladder, promoting blood circulation to remove blood stasis; peicarpium arecae, dredging phlegm and alleviating water retention, guiding Qi downwards; rhizoma bletillae, rhizoma imperatae, stopping bleeding and cooling blood, removing necrotic tissue and promoting granulation; massa medicata fermentata fujianensis, reducing phlegm and helping digestion, strengthening the spleen and stomach; and liquorice, moderating the property of the mentioned herbs.

Prescription analysis of attached powder: in prescription, astragalus membranaceus, tonifying Qi, inducing diuresis to alleviate edema, detoxifying and expelling abscess; angelica sinensis, being warm in nature, enriching blood and nourishing blood; radix paeoniae rubra, enriching blood and invigorating blood circulation, wherein Yin and Yang of two medicines above are united to strengthen the efficacy of enriching blood and invigorating blood circulation; frankincense, promoting qi and invigorating blood circulation, and myrrh, invigorating blood circulation and dissipating stasis, wherein the two medicines above are combined to invigorate blood circulation and dissipate stasis, reduce swelling and alleviate pain, remove necrotic tissue and promote granulation; pseudo-ginseng, invigorating blood circulation and dissipating stasis, stopping bleeding and reducing swelling, whose nature migrates without stagnation; pollen, clearing away heat and promoting secretion of saliva, expelling abscess and eliminating carbuncle; coptis chinensis, capable of distributing to large intestine channel, clearing away heat and promoting dieresis, purging fire and removing toxin.

Efficacy of Decoction 5: strengthening the efficacy of clearing away lung heat and reducing phlegm, relieving swelling and alleviating pain based on description 1 .

Prescription analysis: Principle: herba patriniae, clearing away heat and detoxifying, eliminating carboncle and expelling abscess, dissipating stasis and alleviating pain; houttuynia cordata, being acrid in taste and cold in nature and distributing to lung, clearing away heat and detoxifying, eliminating carbuncle and expelling abscess, inducing diuresis for treating strangurtia. Assistant: Chinese violet, honeysuckle, sargent gloryvine, cooling blood and eliminating carbuncle, clearing away heat and detoxifying, promoting blood circulation and alleviating 
pain, removing periappendicular abscess and stomachache. Complement: semen coicis, Chinese wax gourd kernel, clearing away lung heat and expelling abscess; cortex mori radicis, being acrid scattered, bitter drop, purging lung heat and alleviating water retention; inula flower, descending Qi and reducing phlegm, ventilating lung Qi and eliminating damp; folium eriobotryae, clearing away lung heat and moistening dryness, reducing phlegm and descending Qi, wherein the two medicines above are combined to strengthen the efficacy of ventilating lung Qi and descending Qi; bulbus fritillariae cirrhosae, clearing away lung heat and reducing phlegm, softening hard lumps and dispelling nodes; tangerine pith, activating Qi and reducing phlegm, activating meridians and relieving pain, radix asteris, moistening lung and descending Qi, reducing phlegm and stopping cough, and tussilago farfara, warming lung, moistening lung and reducing phlegm, wherein three medicines above are combined to balance dampness and dryness and strengthen the efficacy of reducing phlegm and activating meridians; radix bupleuri, radix scutellariae, clearing away stagnated fire of liver and gall, harmonizing ShaoYang; cortex moutan, radix paeoniae rubra together with radix bupleuri, radix scutellariae, soothing liver-gallbladder, promoting blood circulation to remove blood stasis; angelica sinensis, nourishing blood, astragalus membranaceus, tonifying Qi, tonifying both Qi and blood to nourish the source of engendering transformation, and liquorice, moderating the property of the mentioned herbs.

Efficacy of Decoction 6: strengthening the efficacy of clearing away lung heat and reducing fire, relieving swelling and alleviating pain based on lung description 1.

Prescription analysis of decoction: Principle: turtle shell, distributing to Yin and removing heat, nourishing Yin and dissipating stagnation, artemisia apiacea, being aromatic and clear, leading pathogen outwards, wherein exiting and entering nourish Yin and remove heat. Assistant: dried radix rehmanniae, nourishing Yin and clearing away heat, helping turtle shell in nourishing Yin and removing deficiency heat. Complement: cortex lycii radicis, radices stellariae dichotomae and blackend swallowwort root, being bitter in taste and cold in nature, cooling blood and removing deficiency heat; rhizome phragmitis, lilium brownii, being sweet in taste and cold in nature, moistening lung and nourishing Yin; semen coicis, Chinese wax gourd kernel and peach kernel, clearing away lung heat and expelling abscess, reducing phlegm and dissipating stasis; platycodon grandiflorum, reducing phlegm and expelling abscess; cortex albiziae, soothing liver and relieving depression, promoting blood circulation and eliminating carbuncle; and liquorice, moderating the property of the mentioned herbs.
Prescription analysis of attached powder: Principle: stir-baked squama manitis, promoting blood circulation and removing stasis, eliminating carbuncle and expelling abscess. Assistant: frankincense, promoting Qi and invigorating blood circulation, and myrrh, invigorating blood circulation and dissipating stasis, wherein the two medicines above are combined to balance Qi and blood, ventilate viscera, circulate meridians, invigorate blood circulation and dissipate stasis, reduce swelling and promote granulation; rhizoma sparganii, being a medicine for regulating Qi in blood, removing blood stasis and activating channels, curcuma zedoary, being a medicine for invigorating blood circulation in Qi, relieving stagnant Qi and removing blood stasis, wherein the two medicines above are combined to enrich both Qi and blood, invigorate blood circulation and dissipate stasis, prom, promote Qi circulation and alleviate pain, dissipate stasis and eliminate lumps. Complement: agelica sinensis, enriching blood and reconciling blood, invigorating blood circulation and alleviating pain, salvia miltiorrhiza, cooling blood and eliminating carbuncle, removing stasis and promoting tissue regeneration. Mediating guide: pericarpium citri reticulatae viride and costustoot, soothing liver and harmonizing stomach, regulating Qi and alleviating pain; massa medicata fermentata fujianensis, helping spleen and stomach to transport, reducing phlegm and removing stagnation; red ginseng, being warm and vigorous, elevating Yang Qi to ascend liver Yang.

Taken together, we may consider that chemotherapy regimens combined with Chinese herbal medicine has greater potential efficacy and lower adverse effects compared with conventional radiation and chemotherapy in the treatment of recurrent or metastatic breast cancer, even when it is in advanced stage.

\section{Conclusion}

In the course of this combined treatment, it had been shown that Chinese herbal medicine played an important role in the therapy of breast mammary carcinoma. Chinese herb might be an additional choice with its better benefits and tolerability in the treatment of recurrent breast mammary carcinoma. Thus there is a potential alternative of chemotherapy tailored to individual patient.

\section{Acknowledgements}

This work was supported by a Grant-in-Aid from the Ministry of Health and Welfare of Nanjing, China (ZKX 2013001), and the Project of Scientific Education and Vitalizing Health Service for Leading Talents and Innovation Teams from the Ministry of Health and Welfare of Jiangsu Province in 2011 (Jiangsu Health Scientific Education [2011] No. 15). We thank Dr XD. Xia for his tech- 
nical assistance during our treatment and kind review of this report.

\section{REFERENCES}

[1] W. Chen, R. Zheng, S. Zhang, P. Zhao, G. Li, L. Wu and J. He, "Report of Incidence and Mortality in China Cancer Registries," Chinese Journal of Cancer ResearchRes, Vol. 25, No. 1, 2013, pp. 10-21.

[2] Z. L. Tang, J. Bai, L. N. Gu, L. Li and D. Xue, “A Systematic Review: Epidemic Status of Prostate Cancer and Breast Cancer from 2000 to 2010 in China," China Cancer, Vol. 22, No. 4, 2013, pp. 260-265.

[3] T. Qin, Z. Yuan, R. Peng, B. Bai, Y. Shi, X. Teng, et al., "HER2-Positive Breast Cancer Patients Receiving Trastuzumab Treatment Obtain Prognosis Comparable with that of HER2-Negative Breast Cancer Patients," Journal of OncoTargets and Therapy, Vol. 9, No. 6, 2013, pp. 341-347. http://dx.doi.org/10.2147/OTT.S40851

[4] X. Li, G. Y. Yang, X. X. Li, Y. Zhang, J. L. Yang, Chang. $\mathrm{J}$, et al., "Bensoussan A. Traditional Chinese Medicine in Cancer Care: A Review of Controlled Clinical Studies Published in Chinese," Plos One, Vol. 8, No. 14, 2013, pp. $1-11$.

[5] K. K. Chan, T. J. Yao and B. Jones, "The Use of Chinese Herbal Medicine to Improve Quality of Life in Women Undergoing Chemotherapy for Ovarian Cancer: a Doubleblind Placebo-Controlled Randomized Trial with Immunological Monitoring," Annals of Oncology, Vol. 22, No. 10, 2011, pp. 2241-2249.

http://dx.doi.org/10.1093/annonc/mdq749

[6] M. Jiang, J. Yang, C. Zhang, B. Liu, K. Chan and H. Cao, "Clinical Studies with Traditional Chinese Medicine in the Past Decade and Future Research and Development,"
Planta Med, Vol. 76, No. 17, 2010, pp. 2048-2064. http://dx.doi.org/10.1055/s-0030-1250456

[7] E. Ernst, "Complementary and Alternative Medicine (CAM) and Cancer: the Kind Face of Complementary Medicine," International Journal of Surgery, Vol. 7, No. 6, 2009, pp. 499-500. http://dx.doi.org/10.1016/j.ijsu.2009.08.005

[8] Q. J. Wang, W. X. Zhu and X. M. Xing, "Analysis of the Incidence and Survival of Female Breast Cancer in Beijing during the Last 20 Years," Chinese Journal of Oncology, Vol. 28, No. 3, 2006, pp. 208-210.

[9] W. Q. Chen, S. W. Zhang, R. S. Zheng, H. M. Zeng, X. N. Zhou, P. Zhao, et al., "Report of Cancer Incidence and Mortality in China, 2009," China Cancer, Vol. 22, No. 1, 2013, pp. 2-12.

[10] N. Klafke, J. A. Eliott, G. A. Wittert and I. N. Olver, "Prevalence and Predictors of Complementary and Alternative Medicine (CAM) Use by Men in Australian Cancer Outpatient Services," Annals of Oncology, Vol. 23, No. 6, 2012, pp. 1571-1578. http://dx.doi.org/10.1093/annonc/mdr521

[11] L. C. Lo., C. Y. Chen., S. T. Chen., H. C. Chen., T. C. Lee and C. S. Chang, "Therapeutic Efficacy of Traditional Chinese Medicine, Shen-Mai San, in Cancer Patients Undergoing Chemotherapy or Radiotherapy: Study Protocol for a Randomized, Double-blind, Placebo-Controlled Trial," Trials, Vol. 13, 2012, p. 232. http://dx.doi.org/10.1186/1745-6215-13-232

[12] I. Cohen, M. Tagliaferri and D. Tripathy, "Traditional Chinese Medicine in the Treatment of Breast Cancer," Seminars in Oncology, Vol. 29, No. 6, 2002, pp. 563-574. http://dx.doi.org/10.1053/sonc.2002.50005 